LA-UR- $94-3552$

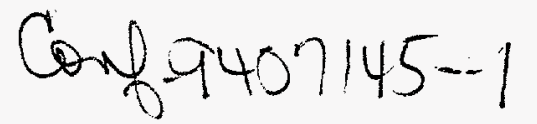

Title: NEUTRON REFLECTIVITY STUDY OF ADSORBED

DIBLOCK COPOLYMERS

Author(s):

G. S. Smith, C. Toprakcioglu, S. M. Baker,J. B. Field, L. Dai, G. Hadziioannou, W. Hamilton, S. Wages

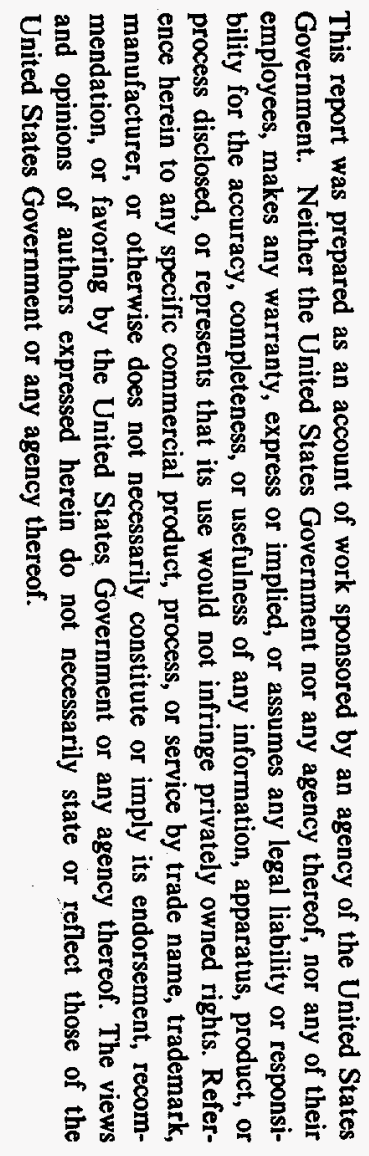

Submitted to:

I1 Nuovo Cimento. Presented at proceedings of Scaling

in Complex Fluids Conference, Catanzaro, Italy, 4-8 JuI 94.

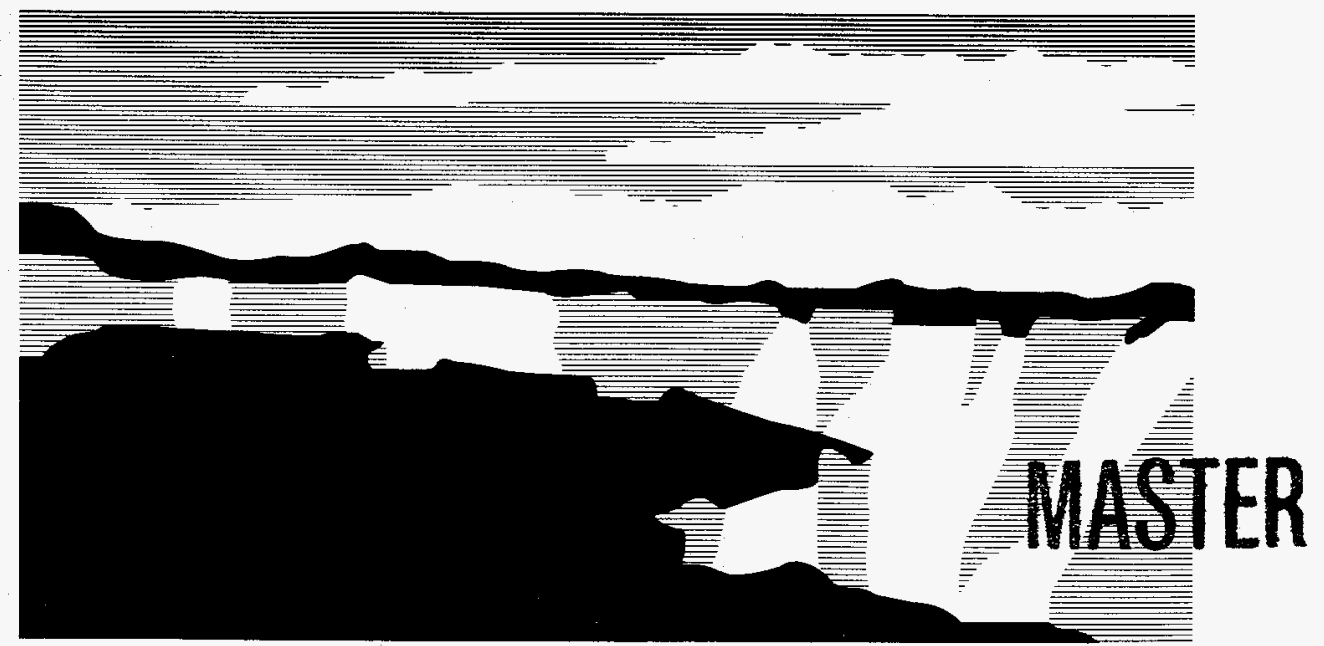

NATIONAL LABORATORY

Los Alamos National Laboratory, an affirmative action/equal opportunity employer, is operated by the University of California for the U.S. Department of Energy under contract W-7405-ENG-36. By acceptance of this article, the publisher recognizes that the U.S. Government retains a nonexclusive, royalty-free license to publish or reproduce the published form of this contribution, or to allow others to do so, for U.S. Government purposes. The Los Alamos National Laboratory requests that the publisher identify this article as work performed under the auspices of the U.S. Department of Energy.

$$
\begin{aligned}
& \text { form No. } 836 \text { R5 } \\
& \text { st } 2629 \text { 10/91 }
\end{aligned}
$$




\section{DISCLAIMER}

Portions of this document may be illegible in electronic image products. Images are produced from the best available original document. 


\title{
Neutron Reflectivity Study of Adsorbed Diblock Copolymers
}

\author{
G.S. Smith 1 , C. Toprakcioglu 2 , S.M. Baker 1,3 , J.B. Field ${ }^{4}$, L. Dai ${ }^{4}$, \\ G. Hadziioannou ${ }^{5}$, W. Hamilton ${ }^{1,6}$ and S. Wages 1
}

1. LANSCE, Los Alamos National Laboratory, Los Alamos, N.M., USA

2. University of Patris, Physics Dept., Patris, Greece

3. Present Address: Dept. of Chemistry, Harvey Mudd College, Claremont, CA, USA

4. Cavendish Laboratory, Cambridge, U.K.

5. Dept. of Polymer Chemistry, University of Groningen, Groningen, Netherlands

6. Present Address: Oak Ridge National Laboratory, Solid State Division, Oak Ridge TN , USA

PACS numbers: $61.12 . \mathrm{Ex}, 61.25 . \mathrm{Hq}, 68.45 .-\mathrm{v}, 68.55 . \mathrm{Jk}$

\begin{abstract}
In this paper, we summarize our cumulative work on neutron reflectivity studies of polystyrene-poly(vinyl-2-pyridine) (PS-PVP) and polystyrenepolyethylene oxide (PS-PEO) adsorbed at a quartz-solvent interface. Deuterated toluene was chosen as the solvent since it is a good solvent for PS and a poor one for either of the other two blocks. In this case, the polystyrene dangles into the solvent while the other block acts as an anchor. The neutron reflectivity studies reveal that the form of the polymer density profile normal to the substrate may be varied from an extended "brush" to a condensed "mushroom" conformation by manipulating the ratio of the molecular weights of the two blocks. In addition, we present new data on the PS-PEO system in a poor solvent, deuterated cyclohexane, under conditions of shear flow in Poiseuille geometry. We find that when the PS-PEO diblock is absorbed from cyclohexane and is allowed to relax, the PS chain takes on a "mushroom" conformation. However, when the shear is applied, the layer shear thickens due to the PS chains extended to nearly twice their original lengths.
\end{abstract}

\section{INTRODUCTION}

The ability of polymer chains to uncoil and stretch into solution make them useful in a number of industrial applications. For example, in making latex paint and various types of ink, particles are coated with polymers to form a "hairy" layer to sterically stabilize these colloidal suspensions[1]. Another place where polymer extension is used is in enhanced separation processes. By attaching binding ligands to polymer chains on glass beads instead of 
directly attaching them to the glass, the effective binding area is increased. Also, once the chains have captured the particle of choice (whether to purify a liquid or to remove precious materials), the polymers may be removed from the beads in order to concentrate the captured moiety. In each of these examples, one may intuitively expect that the effectiveness of these coatings may depend on the conformation of the polymer chains.

The behavior of grafted polymer chains in solution has been the subject of several theoretical studies $[2,3,4,5,6,7,8]$. These studies range from scaling arguments $[2,3,4]$ to mean field theories $[5,6,7]$. The common goal of this work is to understand the behavior of grafted polymers in a good solvent and to extract predictions of the segment density profile as well as scaling laws. Within this body of work, it was found that there exist two regimes for the polymer segment density profiles. If the grafting points are further apart than twice the Flory Radius, $\mathrm{RF}_{\mathrm{F}}$, then the polymer chains don't interact with one another and the predicted shape of the polymers may be thought of as resembling a mushroom. This gives a profile [4] with a density approaching zero near the interface and peaking some distance away from the surface. On the other hand, if the grafting density is high enough that the polymers begin to overlap, then the density profile is predicted to be parabolic [5] with the maximum in the density occurring at the quartz surface and falling to zero in the solvent. In addition to these theoretical studies to understand the static properties of grafted polymers, other theories have been developed for the more complicated, yet more realistic, case of polymer monolayers subjected to shear flow $[8,9,10]$.

Following the work of Tauton[11], we chose to study the segment density profiles of adsorbed diblock copolymers in order to test the theories and gain an understanding of the conformation of grafted polymers in solution. This system is well suited for this task since we may vary the size of either the adsorbed "anchor" block or the stretched soluble blocks. In this way, we can control the polymer surface density to test the relationship between interpolymer spacing and the segment density profile. In order to measure the density profile of the polymeric monolayers at the liquid/solid interface, we use neutron reflectometry $[12,13]$. This technique provides us with a method for measuring the density profile of the polymer monolayer with submolecular resolution. Furthermore, we have recently made modifications to our liquid/solid interface, neutron reflectivity cell [14] so that we can apply a Poiseuille shear flow and study the effects of shear on the polymer's density profile.

In this paper, we summarize our work on the segment density profiles of adsorbed diblock copolymers under static conditions. In addition, we present new data on some of these same materials which shows a dramatic alteration of the polymer conformations when a shear force is applied to the polymer. 


\section{EXPERIMENTAL}

Three different sets of room temperature experiments were performed in this work. In the first set of measurements, the density profiles of polystyrenepolyethylene oxide (PS-PEO) diblock brushes at the deuterated-toluene/quartz interface were measured [12]. In the second set, we measured the density profiles of polystyrene-poly(vinyl-2-pyridine) (PS-PVP) mushrooms at the d-toluene/quartz interface[13]. Finally, in the most recent work, we measured the density profile of PS-PEO in deuterated cyclohexane as a function of shear. In the first two cases, the toluene is a good solvent for the PS and a poorer solvent for the anchor block. In the latter case, while the cyclohexane is a poor solvent for both blocks at room temperature, it is still a better solvent for the PS than the PEO; therefore, the PEO continues to act as anchor and there is still some solvent swelling of the PS block.

In all of the experiments the cells consisted of highly polished quartz blocks (polished to roughly $\lambda / 10$ in flatness). The quartz blocks were sealed against a Teflon block which is machined with a reservoir for the liquid. In the static cell, the solvent/polymer mixture is introduced through glass tubing and sealed with stopcocks $[12,13]$. In the shear cell, a reservoir tank is filled with the solution and then pumped into the space between the Teflon block and the quartz [14]. Once the shear cell is filled, the solvent can be pumped continuously during the experiment so that a shear force is applied to the polymer by the flowing solvent. Extreme care was taken in each case to clean the quartz and the Teflon to avoid any surface contamination. In the static experiments, the quartz was soaked for several hours in $1: 3 \mathrm{HNO}_{3}: \mathrm{H}_{2} \mathrm{SO}_{4}$; then it was rinsed with water, and then it was soaked in $1: 3 \mathrm{HNO}_{3}: \mathrm{HCl}$ for several more hours followed by a distilled water rinse, and finally a methanol rinse. For the shear experiments, the final methanol rinse was replaced with a six hour treatment in a UV-Ozone cleaner and $18 \mathrm{M} \Omega$ water was used for all rinses. The polymer samples were prepared as described previously [15]. The concentrations of the polymer in the solvents were $0.1 \mathrm{mg} / \mathrm{ml}, .05 \mathrm{mg} / \mathrm{ml}$, and $.05 \mathrm{mg} / \mathrm{ml}$ for the PS-PEO brushes, the PS-PVP mushrooms and the PSPEO shear experiments, respectively. In all of the experiments, the polymer was permitted to adsorb to the quartz for at least 6 hours prior to the neutron measurements.

For the static PS-PEO measurements, the neutron reflection experiments were carried out both on the CRISP instrument at the Rutherford Appleton Laboratory and on the SPEAR reflectometer at Los Alamos National Laboratory. The PS-PVP static measurements were carried out on SPEAR and on D17 at the ILL, Grenoble. The PS-PEO shear measurements were carried out only on SPEAR. 


\section{RESULTS AND DISCUSSION}

Figure 1 gives the measured volume fraction profiles for the PS block in PSPVP [13]. In these experiments, the molecular weight of the PS block was fixed at 60,000 while the molecular weight of the PVP block was varied from 30,000 to 120,000 . As shown in Figure 1, the density profiles obtained from the best fits to the reflectivity data are described by Schultz functions which are used to describe the mushrooms[13]. For all three of the PVP molecular weights, the maximum in the PS density profile occurs at roughly the same distance from the quartz interface. Also, the height of the profile simply decreases with increasing molecular weight of the PVP chain while the shape remains the same which shows that the larger anchor blocks decrease the grafting density of the PS blocks. From this data, the absorbance of the polymer on the surface was calculated and the mean spacing between polymers, $s$, was then extracted[13]. In the regime where the Schultz function was appropriate for fitting the data, the molecular weights were found to scale well with $s^{2}$ which is consistent with a description of the system as a flat pancake-shaped PVP block with a constant thickness and a mushroom shaped PS block on top of the PVP. As more mass is added to the PVP block, the pancakes get larger. Since the thickness of the PVP pancake is constant, the increase in PVP volume can only be accommodated by increasing the radius of the pancake. It is also consistent with the notion that the soluble PS blocks are not interacting with each other since they play no role in determining this change in the grafting distances. The value of the maximum in the density profile was also found to scale as $\mathrm{s}^{-2}$. This is consistent with the scaling arguments made for these materials [16].

If we further decrease the size of the anchor block, we see that a Schultz function no longer describes the reflectivity data $[12,13]$. In that case, the data can be fit with a parabolic density profile (Figure 2). A parabolic density profile is predicted by mean field theory [5] for polymer brushes. In the case of a small anchor block size, as we increased the molecular weight of the soluble block we find that the thickness, $\mathrm{L}_{0}$, of the polymer layer scales as $\mathrm{MWPS}^{3 / 5}$. Once again, this is consistent with both the scaling and the mean field theories $[2,3,5]$ considering that the blocks are not chemically bound to the surface, but are free to adjust their spacing [11].

In order to approach the actual conditions to which these materials might be subjected, we have begun to study the density profiles of adsorbed diblock copolymers under shear. In almost any application where these thin films are typically used, a shear force would be applied to the polymer by the solvent. Therefore, we have used our recently designed shear cell[14] to study the effects of shear flow on adsorbed diblock copolymers. We began these neutron reflectivity measurements with the measurements of the static density profile of 184,000 MW PS and 4\% PEO in a poor solvent (cyclohexane 
at room temperature). We found that the reflectivity data could be fit with a Schultz-like function (Figure 3a). Although the previous measurements showed that this diblock was a brush in good solvent it is found to be a mushroom in a poor solvent. This makes sense since we expect $R_{F}$ to be smaller when the polymer is in a poor solvent. The smaller RF allows a cross over from the PS interactions dominating the surface structure to a density profile consistent with dominating inter-anchor interactions (i.e. a mushroom). In contrast, Figure $3 \mathrm{~b}$ shows the fitted volume fraction profile of the same diblock in Figure 3 a sheared at a rate of $\sim 400 \mathrm{~s}^{-1}$. Both profiles include a thin layer which represents the PEO anchor block adsorbed to the quartz surface. Since the area under each curve is the same, one may conclude that there is no addition or removal of material from the surface during shear. Also, as the profiles indicate, the polymer is stretched under shear conditions (i.e. shear thickening occurs). The observed shear thickening is consistent with previous theoretical work on sheared polymer brushes $[8,9]$.

\section{CONCLUSIONS}

We have shown that diblock copolymers are a good system for studying grafting behavior of polymers at a solid/liquid interface. These materials provide us with a tunable system in which we may vary the grafting density by changing the relative sizes of the two polymer blocks. This has allowed us through neutron reflectivity experiments to measure the density profiles of these materials with sub-molecular resolution. We have found that the scaling and mean-field theories [2,3] have validity in their predictions for both the scaling of the $\mathrm{MW}$ and in predicting the functional form of the density profiles in both the mushroom and in the brush regimes. Also, we have presented new data on the effects of shear on the density profile of an adsorbed diblock in a poor solvent. This is the first time that the density profile has been measured with sub-molecular resolution for an adsorbed polymer monolayer under shear flow. We find that while the PS in this particular diblock is in the mushroom regime under static conditions, the PS block is stretched to nearly twice it's static length under applied shear.

\section{ACKNOWLEDGMENTS}

Funding for the SPEAR instrument at the Manuel Lujan Jr., Neutron Scattering Center at Los Alamos National Laboratory is supported by the U.S. Department of Energy, Office of Basic Energy Sciences, and other Department of Energy programs under Contract W-7405-ENG-32 to the University of California. 


\section{REFERENCES}

1. D. H. Napper, "Polymeric Stabilization of Colloidal Dispersions," Academic Press, London, 1983.

2. S. Alexander, J. Phys. (Paris), 38, 983(1977).

3. P.G. de Gennes, J. Phys (Paris), 37, 1445(1976).

4. P.G. de Gennes, Macromolecules, 13, 1069(1980).

5. S.T Milner, T. A. Witten, and M.E. Cates, Macromolecules, 21, 2610(1988).

6. M.D. Whitmore and J. Noolandi, Macromolecules, 23, 3321(1990).

7. M. Muthukumar and Jyh-Shyong HO, Macromolecules, 22,965(1989).

8. J.-L. Barrat, Macromolecules, 25, 832(1992).

9. E.B. Zhulina and T. Pakula, Macromolecules, 25, 754(1992).

10. D.R.M. Williams, Macromolecules, 26, 5806(1993).

11. H. J. Taunton, C. Toprakcioglu, L.J. Fetters, and J. Klein, Macromolecules, 23, 571(1990).

12. J.B. Field, C. Toprakcioglu, R.C. Ball, H. B. Stanley, L. Dai, W. Barford, J. Penfold, G. S. Smith, and W. Hamilton, Macromolecules, 25, 434(1992).

13. J. B. Field, C. Toprakcioglu, L. Dai, G. Hadziioannou, G.S. Smith, and W. Hamilton, J. Phys. II, 2, 2221(1992).

14. S. M. Baker, G.S. Smith, R. Pynn, P. Butler, J. Hayter, W. Hamilton, and L. Magid, Rev. Sci. Instrum., 65 (2), 412(1994).

15. G. Hadziioannou, S. Patel, S. Grannick, and M. Tirrell, J. Am. Chem. Soc., 108, 2869(1986).

16. M.R. Munch and A. P. Gast, J. Chem. Soc. Faraday Trans, 86, 1341(1990). 


\section{Figure Captions}

Figure 1 The fitted volume fraction profiles for the PS-PVP diblock copolymers. The MW of the PS was 60,000 in each case and the MW of the PVP blocks were (a) 30,000 , (b) 60,000 , and (c) 120,000 . The distance $z$ is measured from the quartz interface.

Figure 2 The measured volume fraction profiles for the PS-PEO diblocks in toluene. The molecular weights are given for the PS blocks in the figure where the weight percentages of the PEO blocks are 5.0, 1.5, 4.0, and 0.8 for the $80,000,150,000,184,000$, and 502,000 PS blocks, respectively. The distance $\mathrm{z}$ is measured from the quartz interface.

Figure 3 Results from the neutron reflection measurements on 184,000 PS $/ 4 \%$ PEO adsorbed onto quartz from cyclohexane. When the shear is off (a), the data may be fit with a Schultz-like function [13] which is consistent with the mushroom regime. When the shear is applied at $400 \mathrm{~s}^{-1}$, the PS stretches into the solvent to nearly twice its original length. In this figure, $z=0$ represents the pure solvent interface. The thin step is the PEO anchor block attached to the quartz surface. 


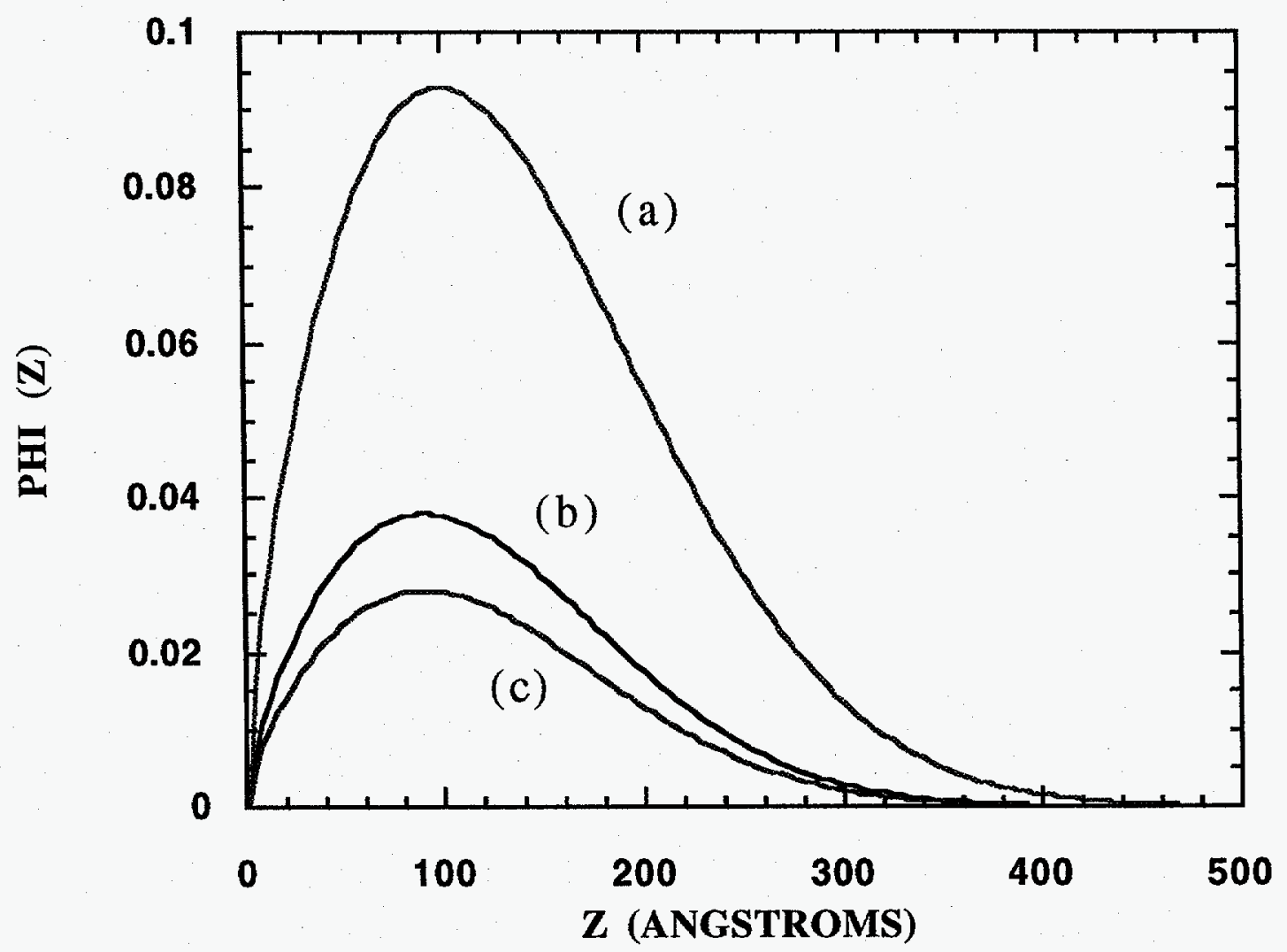

FIGURE 1 


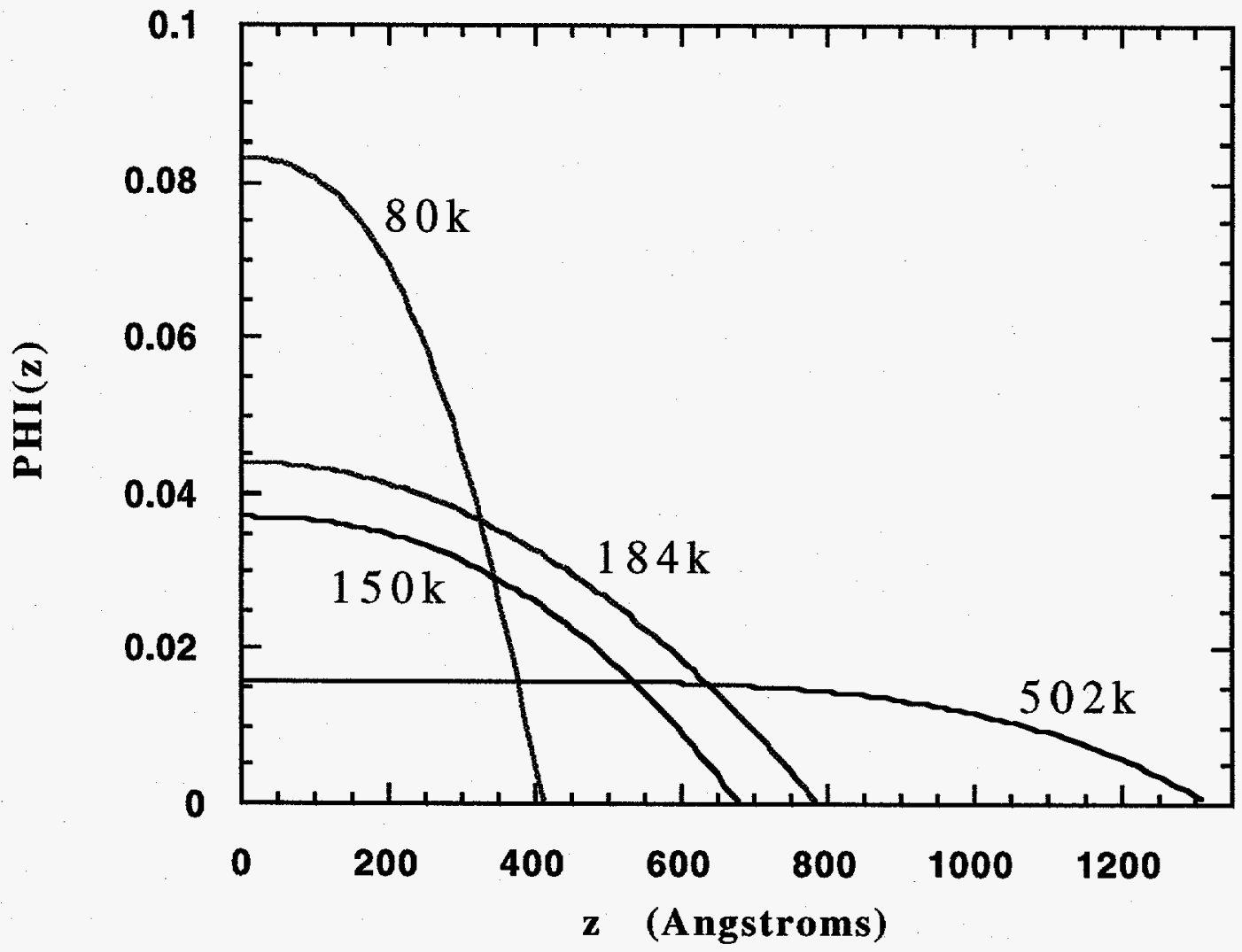

FIGURE 2 


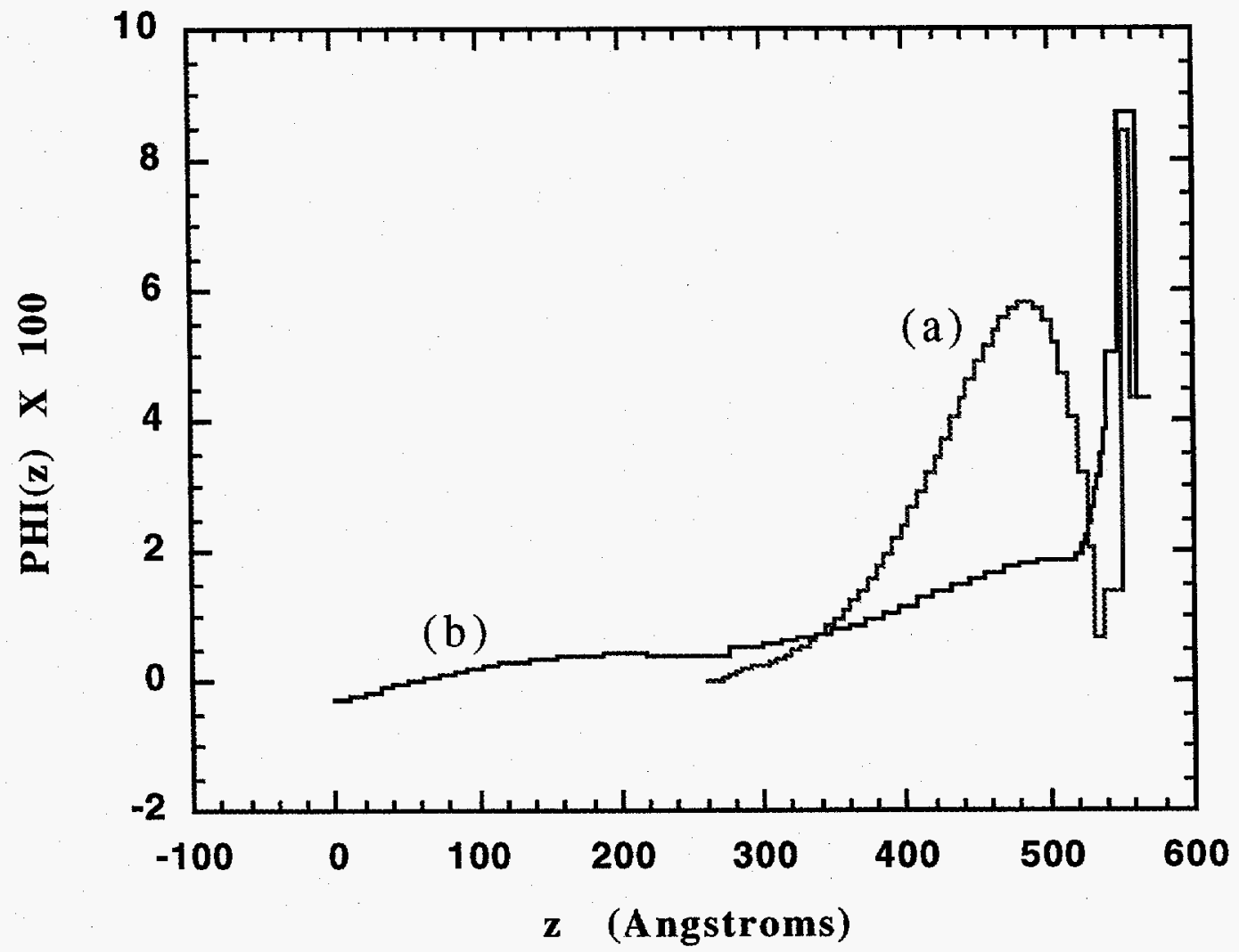

FIGURE 3 